\title{
Multipurpose stabilization of the advanced marine surface crafts
}

\author{
Ruslan A. Sevostyanov ${ }^{1, a}$ and Evgeny I. Veremey ${ }^{1}$ \\ ${ }^{1}$ Saint Petersburg University, Computer Applications and Systems Department, 198504, Saint Petersburg, Russia
}

\begin{abstract}
Advanced marine surface crafts, such as SWATHs, catamarans or hovercrafts become more and more popular for a great range of various tasks. They usually operate at much higher speed than conventional ships. Moreover, in the open sea there are a lot of requirements and restrictions concerning the quality of such crafts' dynamics, especially in case of the wind or waves. This paper considers application of the control law with a special multipurpose structure for autopilot design for amphibious air cushion vehicles. Such control law allows to decompose the autopilot task into simpler optimization subtasks. Efficiency of this approach is shown in the task of stabilizing yaw angle of the air cushion vehicle in the different weather conditions.
\end{abstract}

\section{Introduction}

One of the main goals of creating modern advanced marine crafts is the minimization of the hydrodynamic drag by reduction of the craft's hull area in contact with water. This allows to increase craft's speed and operation range, and to reduce fuel consumption. But there are also some disadvantages, such as increased influence of the wind and waves. Moreover, moving at higher speeds and complicated methods of hull lifting imply stricter requirements and restrictions for craft's dynamics.

In this paper we focus on the particular type of advanced crafts such as air cushion vehicles (ACVs). These crafts are lifted above the supporting surface by the aerostatic pressure created by supplying air into the area below the hull fenced by flexible skirt called air cushion. Due to the air cushion, in general, there is no contact of the rigid hull with the supporting surface which allows ACVs to move over any surface, i.e. ground, water, ice, swamps, etc.

The most complicated condition for ACVs is moving in the open seaway. They work well in case of the calm water, but the presence of wind or waves significantly complicate the operation. This implies the use of more advanced controller for the autopilot design. In this work we propose using the controller of the special structure, called multipurpose control law ([1]) for the task of the stabilization of the ACV's yaw angle. One of the main advantages of such structure is certain flexibility. It allows to comply with several dynamic requirements and restrictions simultaneously by decomposing the main task into simpler independent optimization subtasks. It is also possible to use only necessary parts of the controller in the current moment depending on the environmental conditions. The controller can even be applied for stabilization of some nonlinear systems ([2]).

\section{Multipurpose control law}

Standard stabilization problem is usually considered with respect to the linearized model of the craft's dynamics. Initial nonlinear dynamic model can be written in the following form:

$$
\dot{\boldsymbol{x}}=G(\boldsymbol{x}, \boldsymbol{\delta}, \boldsymbol{d}),
$$

where $\boldsymbol{x} \in E^{\mathrm{n}}$ is the plant state vector, $\boldsymbol{\delta} \in E^{\mathrm{m}}$ - rudders deflections vector, $\boldsymbol{d} \in E^{\mathrm{n}_{\mathrm{d}}}-$ external disturbances vector.

Result of the linearization is the system of linear ordinary differential equations

$$
\begin{aligned}
& \dot{\boldsymbol{x}}=\mathbf{A} \boldsymbol{x}+\mathbf{B} \boldsymbol{\delta}+\mathbf{H} \boldsymbol{d}(t), \\
& \boldsymbol{y}=\mathbf{C} \boldsymbol{x},
\end{aligned}
$$

where $\boldsymbol{x}$ is the vector of the deflections of the states from the equilibrium position, $\mathbf{A}, \mathbf{B}, \mathbf{H}, \mathbf{C}-$ constant matrices.

In addition to system (1) linear model of the rudder actuators should be considered in the form

$$
\dot{\boldsymbol{\delta}}=\boldsymbol{u},
$$

where $\boldsymbol{u} \in E^{\mathrm{m}}$ is the control signal.

The control law for the stabilization of the system (1), (2) has the following structure:

$$
\begin{aligned}
& \dot{\boldsymbol{z}}=\mathbf{A} \boldsymbol{z}+\mathbf{B} \boldsymbol{\delta}+\mathbf{G}(\boldsymbol{y}-\mathbf{C} \boldsymbol{z}), \\
& \xi=F(p)(\boldsymbol{y}-\mathbf{C} \boldsymbol{z}), p=d / d t, \\
& u=\boldsymbol{\mu} \dot{\boldsymbol{z}}+\boldsymbol{v} \boldsymbol{y}+\xi .
\end{aligned}
$$

Here the first equation represents the asymptotic observer. The second one plays role of the dynamic corrector, which can either provide the accurate steering ([3]) or minimize rudders deflections in case of sea waves presence ([4]). Finally, the third one is the control signal for the rudders actuators.

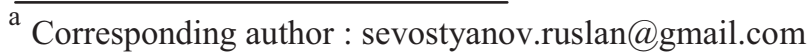


If we represent the dynamic filter in the normal form, then controller (3) can be written as follows:

$$
\begin{aligned}
& \dot{z}=A z+B \delta+G(y-C z), \\
& \dot{p}=\alpha p+\beta(y-C z), \\
& \xi=\gamma p, \\
& u=\mu \dot{z}+v y+\xi .
\end{aligned}
$$

In order to solve the stabilization problem, we have to find coefficients $\boldsymbol{\mu}$ and $\boldsymbol{v}$ of the control signal, matrix $\boldsymbol{G}$ of the asymptotic observer and transfer function $F(p)$ (and, therefore, coefficients $\boldsymbol{\alpha}, \boldsymbol{\beta}$ and $\boldsymbol{\gamma}$ of the normal form) of the corrector. Each of them are usually found through the solution of some optimization problem providing desired quality of the autopilot action.

\section{ACV model}

\subsection{Nonlinear dynamics}

The differential equations of the ACV motion concerning the manoeuvrability with four degrees of freedom can be written as follows $[5,6]$ :

$$
\begin{aligned}
m\left(\dot{V}_{\mathrm{x}}+V_{\mathrm{z}} \omega_{\mathrm{y}}\right) & =F_{\mathrm{xa}}+F_{\mathrm{xh}}+T, \\
m\left(\dot{V}_{\mathrm{z}}-V_{\mathrm{x}} \omega_{\mathrm{y}}\right) & =F_{\mathrm{za}}+F_{\mathrm{zh}}+F_{\mathrm{zc}}+F_{\mathrm{zr}}, \\
I_{\mathrm{x}} \dot{\omega}_{\mathrm{x}} & =M_{\mathrm{xa}}+M_{\mathrm{xh}}+M_{\theta}+M_{\mathrm{xr}}, \\
I_{\mathrm{y}} \dot{\omega}_{\mathrm{y}} & =M_{\mathrm{ya}}+M_{\mathrm{yh}}+M_{\mathrm{yr}},
\end{aligned}
$$

where $\dot{V}_{\mathrm{x}}, \dot{V}_{\mathrm{z}}, \omega_{\mathrm{x}}$ and $\omega_{\mathrm{y}}$ are corresponding linear and angular velocities, $m$ - craft's mass, $I_{\mathrm{x}}$ and $I_{\mathrm{y}}$ moments of inertia. Index "a" denotes aerodynamic profile drag, "h" - hydrodynamic drag, "r" - forces and moments acting on rudders, $F_{\mathrm{zc}}-$ jet force produced by the air blown from the cushion in case of rolling, $M_{\theta}-$ restoring moment and $T$ is the thrust. Corresponding coordinate systems are presented on Fig. 1.

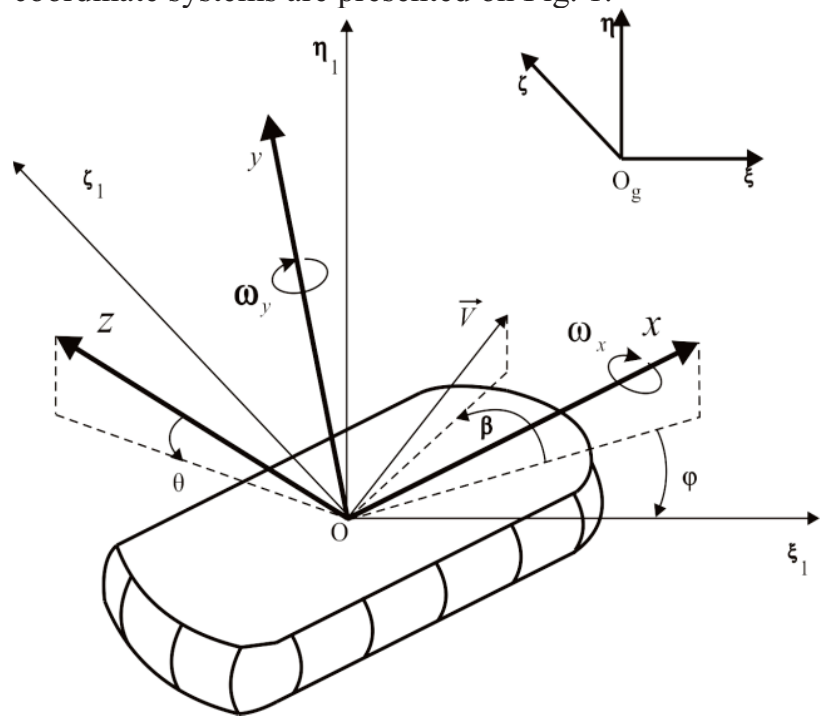

Figure 1. Coordinate systems of the model.

$$
\begin{aligned}
F_{\text {xa }} & =0.5 C_{\text {xa }}\left(\beta_{\mathrm{a}}\right) \rho_{\mathrm{a}} V_{0}^{2} S, \\
F_{\text {za }} & =0.5 C_{\text {za }}\left(\beta_{\mathrm{a}}\right) \rho_{\mathrm{a}} V_{0}^{2} S, \\
M_{\text {xa }} & =0.5 F_{\text {za }} H, \\
M_{\text {ya }} & =0.5\left(C_{\text {mya }}\left(\beta_{\mathrm{a}}\right)+C_{\text {mya }}^{\omega_{\mathrm{y}}} \frac{\omega_{\mathrm{y}} L}{V_{0}}\right) \rho_{\mathrm{a}} V_{0}^{2} S L,
\end{aligned}
$$

where $\beta_{\mathrm{a}}$ is aerodynamic drift angle (with respect to the wind), $\rho_{\mathrm{a}}-$ air density, $V_{0}-$ craft's velocity with respect to the wind, $\mathrm{S}$ - lateral surface area, $H$ - craft's height, $L-$ craft's length. $C_{\mathrm{xa}}\left(\beta_{\mathrm{a}}\right), C_{\mathrm{za}}\left(\beta_{\mathrm{a}}\right)$, $C_{\text {mya }}\left(\beta_{\mathrm{a}}\right), C_{\text {mya }}^{\omega_{\mathrm{y}}}-$ aerodynamic coefficients.

Hydrodynamic drag has the following form:

$$
\begin{aligned}
F_{\mathrm{xh}} & =0.5 C_{\mathrm{xh}}(F r, \beta) \rho_{\mathrm{w}} V^{2} W^{2 / 3}, \\
F_{\mathrm{zh}} & =0.5 C_{\mathrm{zh}}(F r, \beta) \rho_{\mathrm{w}} V^{2} W^{2 / 3}, \\
M_{\mathrm{xh}} & =0.5 F_{\mathrm{zh}} H, \\
M_{\mathrm{yh}} & =0.5\left(C_{\mathrm{myh}}(F r, \beta)+C_{\mathrm{myh}}^{\omega_{\mathrm{y}}}(F r) \frac{\omega_{\mathrm{y}} W^{1 / 3}}{V}\right) \rho_{\mathrm{w}} V^{2} W,
\end{aligned}
$$

where $\beta$ is craft's drift angle, $\rho_{\mathrm{w}}$ - water density, $V$ craft's velocity, $W$ - craft's displacement. $C_{\mathrm{xh}}(F r, \beta)$, $C_{\mathrm{zh}}(F r, \beta), \quad C_{\mathrm{mxh}}(F r, \beta), \quad C_{\mathrm{myh}}(F r, \beta), \quad C_{m y h}^{\omega_{y}}-$ hydrodynamic coefficients. $F r$ is the Froude's number:

$$
F r=\frac{V}{\sqrt{L g}}
$$

Forces and moments acting on rudders are:

$$
\begin{aligned}
F_{\mathrm{zr}} & =C_{\mathrm{r}} \delta \frac{\rho_{\mathrm{a}}}{2} V_{0}^{2} S, \\
M_{\mathrm{yr}} & =F_{\mathrm{zr}} x_{\mathrm{p}}, \\
M_{\mathrm{xr}} & =-F_{\mathrm{zr}} y_{\mathrm{p}}, \\
C_{\mathrm{r}} & =C_{\mathrm{r} 1}\left[\left(1-k_{\mathrm{r}}\right)(1+s) \frac{S_{\mathrm{r}}^{\prime \prime}}{S_{\mathrm{r}}}+\frac{S_{\mathrm{r}}^{\prime}}{S_{\mathrm{r}}}\right], \\
k_{r} & =\frac{1.9 \pi \lambda_{1 \mathrm{r}} s}{\lambda_{\mathrm{r}}\left(1.9 \pi+\lambda_{1 \mathrm{r}}\right)(s+1)}, \\
s & =\sqrt{\frac{1+2.53 T}{\left(\rho_{\mathrm{a}} D^{2} V_{0}^{2}\right)}-1,}
\end{aligned}
$$

where $C_{\mathrm{r}}$ - force coefficient, $\delta$ - rudder angle, $x_{\mathrm{r}}$ and $y_{\mathrm{r}}$ - coordinates of the rudder center, $C_{\mathrm{r} 1}-$ coefficient of the model without thrusters. $S_{\mathrm{r}}-$ rudder area, $S_{\mathrm{r}}^{\prime}, S_{\mathrm{r}}^{\prime \prime}$ - parts of the rudder area inside and outside of the air stream, $\lambda_{\mathrm{r}}, \lambda_{\mathrm{r} 1}$ - coorresponding parts of the rudder length, $D$ - air fan diameter.

Finally, air jet from the cushion and restoring moment are computed as follows:

$$
\begin{aligned}
& F_{\mathrm{zc}}=m g k_{\theta} \theta, \\
& M_{\theta}=-m g h \theta-N_{\theta} \omega_{\mathrm{x}},
\end{aligned}
$$

where $\mathrm{h}$ is metacentric height; $k_{\theta}-$ jet force coefficient; $N_{\theta}$ - restoring coefficient.

Aerodynamic drag has the following form: 


\subsection{Linear model}

Here we linearize system (5) in the neighbourhood of the equilibrium position $V_{\mathrm{x}}=$ const, $\quad V_{\mathrm{z}}=\omega_{\mathrm{y}}=\theta=\delta=0$ assuming that there are no external disturbances. Instead of lateral velocity $V_{\mathrm{z}}$ we're using drift angle $\beta$. In this case $V=V_{\mathrm{x}}=$ const and $\beta=\frac{V_{\mathrm{z}}}{V}$. We're also adding kinematic equation for yaw angle $\varphi$ and linear model of the rudder actuator. Therefore, linear model of the lateral motion of the ACV which is used for the stabilization of the yaw angle has the following form:

$$
\begin{aligned}
\dot{\beta} & =a_{11} \beta+a_{12} \omega_{\mathrm{y}}+b_{1} \delta+h_{1} d(t), \\
\dot{\omega}_{\mathrm{y}} & =a_{21} \beta+a_{22} \omega_{\mathrm{y}}+b_{2} \delta+h_{2} d(t), \\
\dot{\varphi} & =\omega_{\mathrm{y}}, \\
\dot{\delta} & =u .
\end{aligned}
$$

For the ACV with characteristics similar to the Jinsha II-class LCAC [7] weighting 70 tons moving at the speed of $15 \mathrm{~m} / \mathrm{s}$ coefficients in the right parts of the (6) have the following numerical values:

$$
\begin{aligned}
& a_{11}=-0.5718, a_{12}=-1.0, \\
& a_{12}=-0.0083, a_{22}=-0.2828, \\
& b_{1}=-0.0134, b_{2}=-0.0705, \\
& h_{1}=0.043, h_{2}=0.02 .
\end{aligned}
$$

\section{Control law synthesis}

First of all, we derive control signal that stabilizes linear system (6):

$$
u=\mu_{1} \dot{\beta}_{1}+\mu_{2} \dot{\omega}_{\mathrm{y}_{2}}+\mu_{3} \dot{\varphi}_{3}+v \varphi+\xi,
$$

where

$$
\begin{aligned}
& \mu_{1}=-0.1318, \mu_{2}=20.3927, \\
& \mu_{3}=17.4867, v=2.2361 .
\end{aligned}
$$

Next, we construct asymptotic observer assuming that we can measure only the yaw angle:

$$
\begin{aligned}
& \dot{z}_{1}=a_{11} z_{1}+a_{12} z_{2}+b_{1} \delta+g_{1}\left(\varphi-z_{3}\right), \\
& \dot{z}_{2}=a_{21} z_{1}+a_{22} z_{2}+b_{2} \delta+g_{2}\left(\varphi-z_{3}\right), \\
& \dot{z}_{3}=z_{2}+g_{3}\left(\varphi-z_{3}\right),
\end{aligned}
$$

where

$$
g_{1}=24.0296, g_{2}=309.9193, g_{3}=24.8966 \text {. }
$$

Finally, dynamic corrector which plays here the role of the dynamic filter that minimizes rudders deflections under the action of regular waves with frequency 0.65 has the following form:

where

$$
\begin{aligned}
& \dot{p}_{1}=\alpha_{11} p_{1}+\alpha_{12} p_{2}+\beta_{1}\left(\varphi-z_{3}\right), \\
& \dot{p}_{2}=\alpha_{21} p_{1}+\alpha_{22} p_{2}+\beta_{2}\left(\varphi-z_{3}\right), \\
& \xi=\gamma_{1} p_{1}+\gamma_{2} p_{2},
\end{aligned}
$$

$$
\begin{aligned}
& \alpha_{11}=0.0, \alpha_{12}=1.0 \\
& \alpha_{12}=-0.04, \alpha_{22}=-0.4 \\
& \beta_{1}=-3210.8469, \beta_{2}=-4998.4912 \\
& \gamma_{1}=0.0, \gamma_{2}=1.0
\end{aligned}
$$

\section{Simulation results}

Fig. 2 demonstrates craft's turn on 10 degrees without action of wind or waves. In this case dynamic filter (4) is switched off.

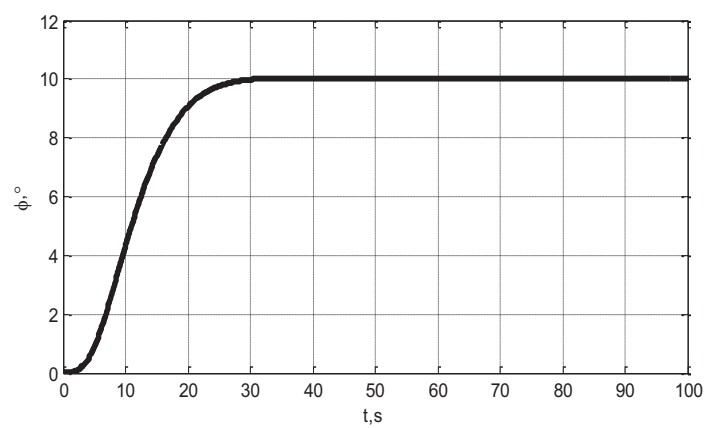

Figure 2. Yaw angle, 10 degrees turn

Fig. 3 demonstrates astatic property of the controller in case of side wind with $10 \mathrm{~m} / \mathrm{s}$ velocity. It can be seen that yaw angle deflects a little more than 0.6 degrees and then converge back to zero, keeping the initial course. Here dynamic filter is also switched off.

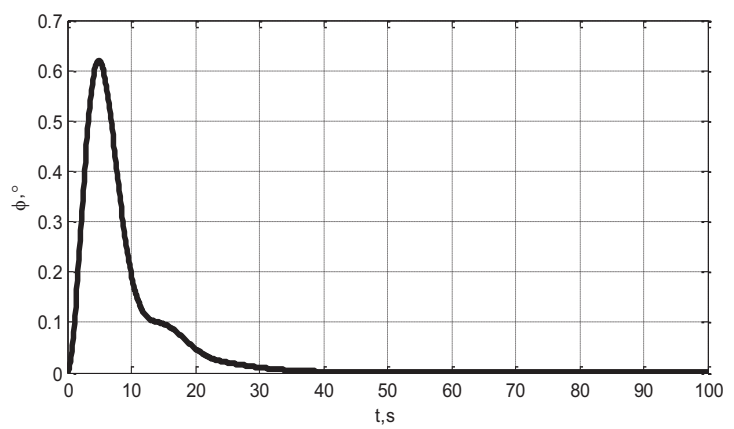

Figure 3. Yaw angle, course keeping under the action of side wind with $10 \mathrm{~m} / \mathrm{s}$ velocity.

Finally, Fig. 4-5 demonstrate the craft's dynamics in case of regular side waves with 4-th number intensity on the Beaufort scale:

$$
d(t)=0.2 \sin (0.65 t)+0.1 \sin (0.55 t)+0.1 \sin (0.75 t) .
$$

Here the dynamic filter was switched on only after 300 seconds. It can be seen that there's no big difference in the yaw dynamics before and after switching the filter (Fig. 4), but the rudders action after 300 seconds decreases significantly (Fig. 5).

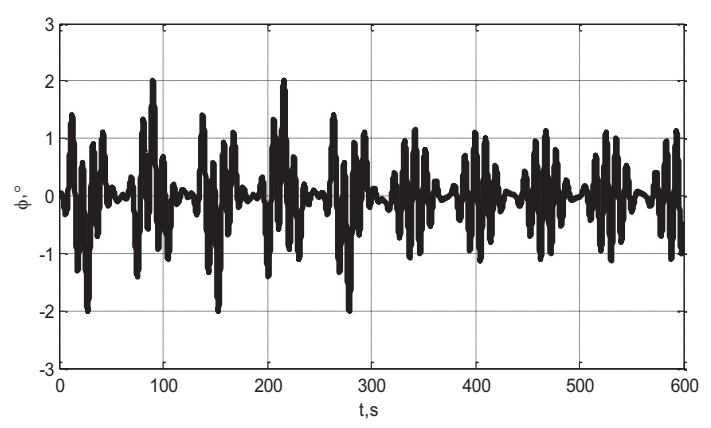

Figure 4. Yaw angle, course keeping under the action of regular side waves. 


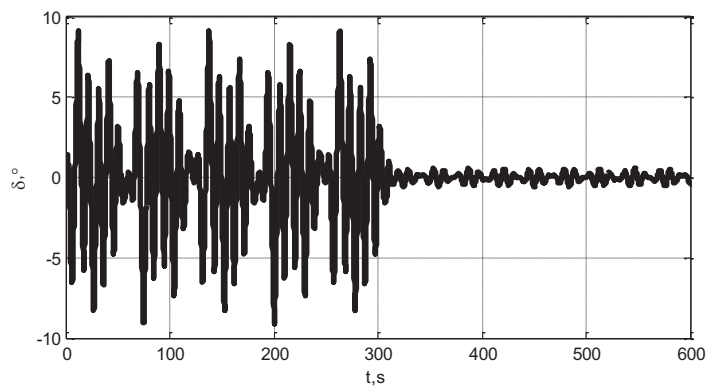

Figure 5. Rudders' deflections, course keeping under the action of regular side waves.

\section{Conclusion}

The goal of this work is to show the efficiency of the multipurpose control law in the task of the stabilization of the course angle of the particular type of advanced marine surface crafts such as air cushion vehicle. It can be seen from previous section that such controller actually stabilizes the course angle even in presence of the wind or waves.

Besides, the structure of the control law has certain flexibility. Each requirement for the control quality can be isolated and solved as independent task. Depending on the current environmental conditions some parts of the controller can be switched off for computational economy and overall control efficiency.

\section{References}

1. E. I. Veremey, J. Marine Sci. Appl., 13, 127 (2014)

2. E. I. Veremey, Dynamical Correction of Positioning Control Laws, Proceedings of 9th IFAC Conference on Maneuvering and Control of Marine Craft, 31 (2012)

3. E. I. Veremey, M. V. Korovkin, M. V. Sotnikova, Ships' Steering in Accurate Regime Using Autopilot with Special Structure of Control Law, IFACPapersOnLine, 48 (16), 7 (2015)

4. E. I. Veremey, Optim. Control Appl. Meth. (2015)

5. L. Yun, A. Bliault, Theory and Design of Air Cushion Craft, (London: Arnolds, 2000)

6. Y. I. Voytkunskiy, Ship Theory Manual, 3 (Leningrad: Sudostroyeniye, 1985) (In Russian)

7. S. Saunders, Jane's fighting ships, 2003-2004, Coulsdon, Surrey, UK; Alexandria, (VA: Jane's Information Group, 2003) 